\title{
Das Vorwort
}

Der vorliegenden Studie liegt das Forschungsprojekt zugrunde, das ich im Frühjahr 2007 am Institut für Systematische Theologie an der Evangelischen Theologischen Fakultät der Martin-Luther-Universität Halle-Wittenberg begonnen und im Herbst 2015 in Berlin abgeschlossen habe. Der zweite Hauptteil dieser Studie - unter dem Titel Religion und Individualität. Eine Untersuchung zur Philosophischen Ethik Schleiermachers - wurde im Sommersemester 2016 von der Theologischen Fakultät der Humboldt-Universität zu Berlin als Dissertation zum Dr. phil. angenommen. Aufgrund der hiesigen Bestimmungen zum Umfang der Dissertation konnte der erste Hauptteil seinerzeit nicht eingereicht werden. Für die Veröffentlichung wurde die gesamte Studie durchgesehen und überarbeitet. Die nach dem Abschluss dieser Studie erschienene Literatur konnte nur zum Teil berücksichtigt werden.

Religion und Individualität sind zwei Themen, die mich bereits während meines Studiums der Philosophie und Religionswissenschaft in China sehr beschäftigt haben. Daher war es eine große Freude für mich als junge Wissenschaftlerin, das Verhältnis von Religion und Individualität bei Friedrich Schleiermacher, einem der bedeutendsten Autoren in der europäischen Theologie- und Philosophiegeschichte, untersuchen zu können. An dieser Stelle möchte ich mich zuallererst bei Herrn Prof. Dr. Ulrich Barth (Halle) bedanken. Er hat mein wissenschaftliches Interesse erkannt, mir das Thema zur Promotion vorgeschlagen und das Projekt in dessen Vorbereitungsphase und Anfangsphase mit großem Engagement betreut. Ihm habe ich auch für zahlreiche Vorlesungen und Seminare im Fachbereich Systematische Theologie und Religionsphilosophie zu danken. Sie ermöglichten es mir, eine solide Grundlage für die Studie zu Schleiermacher zu gewinnen.

Von Herzen gedankt sei ebenfalls Herrn Prof. Dr. Roderich Barth (Leipzig). Er hat meinen bisherigen akademischen Werdegang in Deutschland und dieses Forschungsprojekt in allen Phasen mit Rat und Tat unterstützt, mich selbstständig forschen lassen und mich nicht zuletzt immer wieder angeregt, die langjährige Studie trotz mancher offener Fragen abzuschließen. Meinen herzlichen Dank möchte ich auch den Mitgliedern des von Herrn Ulrich Barth und von seinem damaligen wissenschaftlichen Mitarbeiter Herrn Roderich Barth geleiteten halleschen Doktorandenkolloqiums für stetige freundschaftlich-konstruktive Begleitung ausdrücklich aussprechen: Dr. Christof Ellsiepen, Prof. Dr. Andreas Kubik-Boltres, Prof. Dr. Christopher Zarnow, PD Dr. Björn Pecina, PD Dr. Georg Neugebauer, Dr. Marianne Schröter, Dr. Friedrich Ley, PD Dr. Martin Fritz, PD Dr. Roland Lehmann, Stefan Egenberger, Stephan Feldmann, Dr. Georg Raatz, Dr. Lars Heinemann, Dr. Martin Gröger, Bradley Schmidt, Christian Buro, Anne-Maren Richter und Dr. Constantin Plaul. Über Jahre hinweg habe ich durch ihre Forschungsprojekte viel gelernt, fachlich wie sprachlich. Besonderer Dank gilt denjenigen Kolleginnen und Kollegen, die an 
dem Korrekturlesen meiner Arbeit in ihren unterschiedlichen Stadien beteiligt waren und dabei mit Hinweisen, Anfragen und Anregungen nicht gezögert haben. Ohne die Begleitung dieser einzigartigen wissenschaftlichen Geselligkeit wäre das Zustandekommen der vorliegenden Studie nicht möglich gewesen. Mein herzlicher Dank richtet sich außerdem an Herrn Prof. Dr. Jörg Dierken (Halle), der mich während meiner Studienzeit in der Saalestadt freundlich gefördert hat.

Nach Halle wurde die Theologische Fakultät der Humboldt-Universität zu Berlin die zweite Station meiner akademischen Ausbildung in Deutschland. Herrn Prof. Dr. Andreas Arndt, Herrn Prof. Dr. Wilhelm Gräb und Herrn Prof. Dr. Notger Slenczka danke ich dafür herzlich, dass ich mit dem zweiten Hauptteil der vorliegenden Studie promoviert wurde. Herr Gräb hat dieses Forschungsprojekt nicht erst in dessen Schlussphase in Berlin tatkräftig unterstützt, sondern es bereits zuvor mit fachlichem Interesse und wertvollen Ratschlägen begleitet. Zahlreiche Gespräche mit Herrn Arndt über verschiedene Probleme während der Arbeit und über eigene Zweifel an dem Projekt haben mir nicht nur einen neuen Zugang zu Schleiermacher eröffnet, sondern mir auch dabei geholfen, eine realistische Haltung zur Wissenschaft $\mathrm{zu}$ bilden, nämlich dass alle Forschungen nur Zwischenergebnisse sein können. Herr Gräb und Herr Arndt haben darüber hinaus die Mühen des Erst- und Zweitgutachtens für die Dissertation auf sich genommen. Auch hierfür weiß ich mich ihnen zu besonderem Dank verpflichtet. Herr Prof. Dr. Andreas Feldtkeller hat mir während der Phase, in der ich diese Studie für die Veröffentlichung überarbeitete und mich zugleich auf ein neues Forschungsprojekt vorbereitete, in vielerlei Hinsicht beigestanden. Ihm sei auch herzlich Dank gesagt.

Über die Universitäten hinaus habe ich für dieses Forschungsprojekt viel Förderung in der Wissenschaft bekommen. Dafür bedanke ich mich zunächst bei der Internationalen Schleiermacher-Gesellschaft unter der Leitung von Herrn Prof. Dr. Ulrich Barth und Herrn Prof. Dr. Jörg Dierken. Einige Abschnitte dieser Studie konnten im Rahmen des alljährlichen Wittenberger Schleiermacher-Symposiums vorgestellt werden. Für die freundliche Begleitung und für die aufmerksamen und kritischen Anfragen und Anregungen möchte ich vor allem Herrn Dr. Hermann Patsch (München), Herrn Prof. Dr. Martin Ohst (Wuppertal), Herrn Prof. Dr. Folkart Wittekind (Essen), Frau Dr. Christiane Ehrhardt (Berlin), Herrn PD Dr. Simon Gerber (Berlin) und Herrn PD Dr. Peter Grove (Århus) danken. Mein besonderer Dank gilt auch der Schleiermacher-Forschungsstelle der Berlin-Brandenburgischen Akademie der Wissenschaften. In der Schlussphase dieses Projekts und bei der Vorbereitung auf die Veröffentlichung haben Herr Dr. Wolfgang Virmond ( $\dagger$ ), Frau Dr. Sarah Schmidt und Herr Dr. Holden Kelm mich stets freundlich beraten.

Ohne die finanzielle Unterstützung wäre ein solches Forschungsprojekt nicht vorstellbar. $\mathrm{Zu}$ tiefem Dank verpflichtet bin ich in erster Linie dem Evangelischen Entwicklungsdienst (EED), der mein Promotionsstudium in der Evangelischen Theologie in Halle und diese Studie jahrelang großzügig förderte. Finanzielle Unterstützung erfuhr ich danach von der Mission EineWelt in Neuendettelsau, und zeitweise 
bekam ich auch Stipendien von Lunds Missionssällskap (Schweden) und von der Stiftung Areopagos (Norwegen). Für den Abschluss der Promotion in Berlin wurde mir das Caroline-von-Humboldt-Stipendium verliehen. Ihnen allen danke ich herzlich.

Zum Gelingen dieses Forschungsprojekts haben darüber hinaus eine Reihe weiterer Institutionen und Personen auf unterschiedliche Weise beigetragen, und auch bei denen möchte ich mich an dieser Stelle herzlich bedanken. Ich danke meinem $\mathrm{zu}$ früh verstorbenen Lehrer Herrn Prof. Xian Zhang an der Sun Yat-Sen Universität (Guangzhou) dafür, dass er mein wissenschaftliches Interesse an Schleiermacher bereits früh förderte und mich ermutigte, meine akademische Ausbildung in Deutschland fortzusetzen. Ich danke dem Institute of Sino-Christian Studies (Hong Kong) unter der Leitung von Herrn Daniel H. N. Yeung für treue Wegbegleitung und vielseitige Unterstützung. Ich danke Frau Gesine Mattel-Pegam (Bochum) dafür, dass sie mich in der Anfangsphase dieses Projekts beim wissenschaftlichen Schreiben in deutscher Sprache intensiv förderte. Ich danke Frau Dr. Renate Anders, der ehemaligen Studienleiterin der Theologischen Fakultät in Halle, dafür, dass sie meinen Weg in Deutschland stets hilfreich begleitet hat. Ich danke der Zweigbibliothek Theologie und der Zweigbibliothek Europäische Aufklärung an der Martin-LutherUniversität Halle-Wittenberg für freundliche Unterstützung. Ich danke dem Haus der Stille im Evangelischen Zentrum Kloster Drübeck dafür, dass es mir zwischen den intensiven Arbeitsphasen einen Rückzugsort zur Erholung anbot.

Für die Aufnahme der gesamten Studie in die Reihe „Schleiermacher-Archiv“ danke ich ihren Herausgebern herzlich. Herr Dr. Hermann Patsch hat die Studie für die Veröffentlichung gründlich durchgesehen. Der Dank an ihn liegt mir besonders am Herzen. Herrn René Colome (Berlin) danke ich für die technische Hilfe bei der Manuskripterstellung. Herr Jakob Simon, Herr Johann Gartlinger und Herr Jan Philipp Hahn haben die mühsame Arbeit der orthographischen Abschlusskorrektur übernommen, Frau Marianne Wegner hat das Personenregister erstellt. Ihnen allen sage ich gleichfalls herzlichen Dank. Für die Betreuung seitens des Verlages danke ich Herrn Dr. Albrecht Döhnert, Frau Dr. Eva Frantz und Herrn Lukas Lehmann von Herzen. Die Veröffentlichung wurde durch einen Druckkostenzuschuss der Evangelischen Kirche in Deutschland (EKD) und der Union Evangelischer Kirchen (UEK) unterstützt.

Mein besonders herzlicher Dank gilt meiner Familie - meiner Mutter Youqun Yi, meinem Vater Keyu Jiang, meiner Schwester Manshu Jiang und meinem Bruder Xueyu Jiang. Sie haben mich auf meinem außergewöhnlichen Lebensweg nicht nur bedingungslos und vertrauensvoll unterstützt, sondern sie haben mir über viele Jahre hinweg auch stets gezeigt, dass man den Mut für das Leben nie verlieren soll. Diese Zuversicht hat mir während der schwierigen Arbeitsphase dieser Studie sehr geholfen. Die vielen Freunde, die mir im Laufe der langjährigen Arbeit oft auf unterschiedliche Weisen beigestanden und geholfen haben, kann ich hier nicht einzeln nennen. Ihnen gegenüber empfinde ich ebenfalls eine tiefe Dankbarkeit. 
Dass die vorliegende Untersuchung zu Schleiermacher entstanden ist und abgeschlossen werden konnte, habe ich schließlich Herrn Prof. Dr. Theodor Holzdeppe Jørgensen, zu Lebzeiten Professor für Systematische Theologie an der Universität Kopenhagen, und seiner Frau Lise Jørgensen zu danken. Herr Jørgensen hat dieses Forschungsprojekt in allen seinen Höhen und Tiefen mit großem fachlichem Interesse begleitet und mich unermüdlich angespornt. Als Erster hat er den Entwurf dieser Studie im Frühsommer 2015 gelesen und mit kritischen Anmerkungen versehen. Nicht zuletzt führt mein wissenschaftliches Interesse an Schleiermacher auf einen Gastvortrag zurück, den er während meines Studiums, im Herbst 1999, in Guangzhou hielt. Das war meine erste Begegnung mit diesem großen deutschen Gelehrten. Herrn Jørgensen sei das Buch gewidmet.

Berlin, April 2020 Manke Jiang (蔣漫軻) 